HEALTHCARE DELIVERY

\title{
Basic and comprehensive emergency obstetric and neonatal care in 12 South African health districts
}

\author{
R C Pattinson, J D Makin, Y Pillay, N van den Broek, J Moodley \\ Bob Pattinson is Director of the South African Medical Research Council Maternal and Infant Health Care Strategies Unit, Department of \\ Obstetrics and Gynaecology, Faculty of Health Sciences, University of Pretoria, South Africa, and Jenny Makin is the epidemiologist on the team. \\ Yogan Pillay is Deputy Director-General for Programmes in the National Department of Health, and Nynke van den Broek is Professor and Head \\ of the Maternal and Child Health Department of the Liverpool School of Tropical Medicine, UK. Jack Moodley is chairman of both the Emergency \\ Obstetric Simulation Training Board and the National Committee for Confidential Enquiries into Maternal Deaths in South Africa.
}

Corresponding author: $R$ C Pattinson (robert.pattinson@up.ac.za)

\begin{abstract}
Aim. To assess the functionality of healthcare facilities with respect to providing the signal functions of basic and comprehensive emergency obstetric care in 12 districts.

Setting. Twelve districts were selected from the 52 districts in South Africa, based on the number of maternal deaths, the institutional maternal mortality ratio and the stillbirth rate for the district.

Methods. All community health centres (CHCs) and district, regional and tertiary hospitals were visited and detailed information was obtained on the ability of the facility to perform the basic (BEmONC) and comprehensive (CEmONC) emergency obstetric and neonatal care signal functions.

Results. Fifty-three CHCs, 63 district hospitals (DHs), 13 regional hospitals and 4 tertiary hospitals were assessed. None of the CHCs could perform all seven BEmONC signal functions; the majority could not give parenteral antibiotics (68\%), perform manual removal of the placenta (58\%), do an assisted delivery (98\%) or perform manual vacuum aspiration of the uterus in a woman with an uncomplicated incomplete miscarriage (96\%). Seventeen per cent of CHCs could not bag-and-mask ventilate a neonate. Less than half (48\%) of the DHs could perform all nine CEmONC signal functions ( $81 \%$ could perform eight of the nine functions), $24 \%$ could not perform caesarean sections, and $30 \%$ could not perform assisted deliveries.

Conclusions. The ability of the CHCs and district hospitals to perform the signal functions (lifesaving services) of basic and comprehensive emergency obstetric care was poor in many of the districts studied. This implies that safe maternity care was not consistently available at many facilities conducting births.
\end{abstract}

S Afr Med J 2015;105(4):256-260. DOI:10.7196/SAMJ.9181

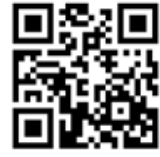

The South African (SA) Rapid Mortality Surveillance Report of $2012^{[1]}$ concluded that 'There is an urgent need to review possible interventions to further reduce maternal and child mortality if the MDG [Millennium Development Goals] targets are to be

met by 2015

Complications of HIV infection as reflected in maternal deaths due to non-pregnancy-related infections are the most common underlying cause of maternal death in SA. ${ }^{[2]}$ There has been a massive effort by the National Department of Health $(\mathrm{NDoH})$ to screen and treat pregnant women who are HIV-infected, and this effort is beginning to show signs of success. The institutional maternal mortality ratio (iMMR) has decreased from the 2011 - 2013 sixth Saving Mothers report, mainly due to the drop in maternal deaths due to non-pregnancy-related infections. Ninety-five per cent of these women were HIV-infected. ${ }^{[2]}$ The infant mortality rate has dropped dramatically, as reported by Dorrington et al..$^{[1]}$ Screening and treating pregnant women for HIV infection remains the highest priority; however, although non-pregnancy-related infections accounted for $40 \%$ of deaths in $2011-2013,{ }^{[2]}$ other causes still accounted for $60 \%$ of maternal deaths. The iMMR for direct causes of maternal death has remained the same for the past decade. ${ }^{[2]}$ This is particularly disappointing given the efforts on the part of the $\mathrm{NDoH}$ and the National Committee for Confidential Enquiries into Maternal Deaths in South Africa (NCCEMD) to provide information such as guidelines and protocols and to give all healthcare providers involved in maternity care this information.

Complications in pregnancy and labour can occur even in the best of circumstances. Many women who develop complications have one or more detectable risk factors, and complications can be anticipated. However, the majority of women who have risk factors do not develop a serious problem, ${ }^{[3]}$ i.e. the risk factors are not very specific. Most importantly, a large proportion of serious complications occur among women with no recognisable risk factors at all, ${ }^{[4]}$ and until the complication occurred they were regarded as having low-risk pregnancies. For these reasons attempts need to be directed to preventing death once the complication has occurred. The sooner a complication is recognised and treated, the better the outcome. Most pregnant women in SA ( $60 \%)$ give birth at the primary level of care, namely in community health centres (CHCs) and district hospitals (DHs).

If an impact on the iMMR is to be made, recognition, stabilisation and treatment or referral of the obstetric emergency must occur at the site closest to where the complication occurred. There are three essential factors:

- Healthcare providers with sufficient knowledge and skills to recognise, stabilise and treat or refer the patient

- Healthcare facilities with the essential lifesaving services available, such as being able to perform caesarean sections (CSs)

- An efficient interfacility transfer system. 
A way to reduce these deaths rapidly is by improving emergency obstetric care. Kerber ${ }^{[5]}$ using the Lives Saved Tool ${ }^{[6]}$ estimated that approximately 9000 maternal and perinatal deaths in SA could be averted if comprehensive emergency obstetric and neonatal care (CEmONC) was fully implemented.

The emergency obstetric care package is a list of lifesaving services or 'signal functions' that define a health facility with regard to its capacity to treat obstetric and neonatal emergencies. Developed by the World Health Organization (WHO) and agreed on internationally by all United Nations organisations, it was first developed and tested in 1992, published as guidelines for monitoring the availability and use of obstetric services issued by UNICEF, the WHO and UNFPA, reviewed and modified in 2006, and published by the WHO as a handbook in 2009. ${ }^{[7]}$ There are seven basic emergency care (BEmONC) signal functions and nine CEmONC signal functions (BEmONC and two others) (Table 1).

A rapid drop in mortality can be achieved by ensuring that these lifesaving services are available, correctly used and accessible to the community. ${ }^{[7]}$ Each lifesaving service, as measured by the signal functions, is important in maternal and neonatal care at facility level. These signal functions, which are easily measured, are markers of these lifesaving services and assessing them gives an indication of the ability of a particular facility to provide emergency obstetric care when complications occur during pregnancy, birth or the postpartum period. This knowledge can be used to identify gaps in availability of essential obstetric care and to catalyse the changes necessary to improve the service.

This survey was undertaken at the start of the Essential Steps in Managing Obstetric Emergencies and Emergency Obstetric Simulation Training (ESMOE-EOST) programmes to establish the functionality of $\mathrm{CHCs}$ and district, regional and tertiary hospitals in 12 health districts in SA with respect to emergency obstetric and neonatal care by assessing these signal functions. The ESMOE-EOST programmes improve the knowledge and skills of healthcare providers in managing obstetric and neonatal emergencies $^{[8]}$ and are being introduced at scale to all the districts in SA.

\section{Methods}

This survey was performed between July and October 2012. In all, 133 health institutions were visited (53 CHCs, $63 \mathrm{DHs}$, 13 regional hospitals (RHs) and 4 provincial tertiary hospitals). A data monitoring team

Table 1. Signal functions used to identify basic and comprehensive emergency obstetric care services ${ }^{[15]^{*}}$

\begin{tabular}{ll}
\hline BEmONC services & CEmONC services \\
\hline 1. Administer parenteral antibiotics & Perform signal functions 1 - 7, plus: \\
2. Administer parenteral uterotonic drugs (i.e. & 8. Perform CS \\
oxytocin) & 9. Provide blood transfusion \\
3. Administer parenteral anticonvulsants for pre- & \\
eclampsia and eclampsia (i.e. magnesium sulphate) & \\
4. Manual removal of retained placenta & \\
5. Remove retained products of conception (e.g. & \\
manual vacuum aspiration) & \\
6. Perform assisted vaginal delivery (e.g. vacuum & \\
delivery) & \\
7. Perform basic neonatal resuscitation with bag and \\
mask
\end{tabular}

\section{Table 2. Details of scoring system}

\begin{tabular}{ll}
\hline Score MMR & $1=180-230 ; 2=230-280 ; 3=>280 / 100000$ live births \\
Score SBR & $1=25-27 ; 2=>27 / 1000$ births \\
Score number of MDs & $1=100-150 ; 2=150-200 ; 3=>200$ \\
Score province & $2=$ highest MMR; $1=$ second-highest MMR \\
MDs = maternal deaths. &
\end{tabular}

Table 3. Distribution of number of signal functions of emergency obstetric care available by type of healthcare facility assessed

\begin{tabular}{|c|c|c|c|}
\hline Number of signal functions & $\begin{array}{l}\text { CHCs }(N=53) \\
n(\%)\end{array}$ & $\begin{array}{l}\text { DHs }(N=63) \\
n(\%)\end{array}$ & $\begin{array}{l}\text { RHs and tertiary hospitals } \\
(N=17) \\
n(\%)\end{array}$ \\
\hline \multicolumn{4}{|l|}{ CEmONC } \\
\hline Perform all 9 functions & NA & $30(47.6)$ & $15(88.2)$ \\
\hline Perform 8 functions & NA & $21(33.3)$ & $2(11.8)$ \\
\hline \multicolumn{4}{|l|}{ BEmONC } \\
\hline Perform all 7 functions & $0(0.0)$ & $7(11.1)$ & - \\
\hline Perform 6 functions & $3(5.7)$ & $3(4.8)$ & - \\
\hline Perform 5 functions & $24(45.3)$ & $2(3.2)$ & - \\
\hline Perform 4 functions & $12(22.6)$ & - & - \\
\hline Perform 3 functions & $11(20.8)$ & - & - \\
\hline Perform 2 functions & $3(5.7)$ & - & - \\
\hline Perform 1 function & - & - & - \\
\hline $\mathrm{NA}=$ not applicable. & & & \\
\hline
\end{tabular}

of between three and six members visited each of the sites. The team consisted of members of the data monitoring team of the South African Medical Research Council (MRC) Maternal and Infant Health Care Strategies Unit and various members of the local or provincial maternal and child health units. Before the visit, a planning meeting was held with the district and provincial managers and the purpose and the baseline survey form were explained. Each site then completed the form prior to the visit by the data monitoring team. The objective of the baseline survey was to describe each of the sites in terms of their functionality with respect to the signal functions required for basic and comprehensive emergency care. Information on the referral system and resources regarding the signal function was collected at each site. 
The standard programme for the visit, after the team had introduced themselves to the CEO and hospital or CHC management, was a workshop explaining the visit followed by training on the new national birth register and the monthly data sheets and a walk through the site to confirm the data entered on the baseline survey form and ensure that all questions were answered and comments recorded.

The data were entered by two data enterers at the MRC unit. Some survey forms were entered twice for quality assurance. The data were cleaned, and sites where there were incongruences were contacted again to verify the information.

\section{Selection of districts}

Twelve districts were selected, using a scoring system developed specifically for this programme and using each district's iMMR, stillbirth rate (SBR), and number of maternal deaths from 2008 to 2010 An additional 2 points were given for the worst-performing and 1 point for the second-worst-performing district in the province. This was done to ensure some parity between the provinces. The scoring system is shown in Table 2. Districts scoring $\geq 5$ were used. The numbers of births and stillbirths per district were obtained from the District Health Information System and the numbers of maternal deaths per district from the NCCEMD database. Data from 2008 to 2010 were used. The 12 districts identified contributed to half of all maternal deaths occurring outside districts with medical schools in SA during 2008 . 2010. Districts with medical schools receive referrals from neighbouring districts, which may contribute to a higher score, and were therefore excluded.

\section{Ethics}

The CEO of every site and the district manager of each district gave permission for the survey, which was approved by the Ethics Committee of the Faculty of Health Sciences, University of Pretoria.

\section{Results}

Table 3 gives the number of signal functions that the various levels of care could provide and Table 4 the availability and ability of each individual function at the levels of care. All CHCs were able to give oxytocics and anticonvulsants, but only $32 \%$ could give parenteral antibiotics. While all CHCs could give magnesium sulphate, only $48 \%$ had a patella hammer available to test reflexes. Nine DHs (14\%) similarly lacked a patella hammer but could provide magnesium sulphate.

Table 4. Summary of availability of signal functions of emergency obstetric care

\begin{tabular}{|c|c|c|c|}
\hline Perform signal function & $\begin{array}{l}\text { CHCs }(N=53) \\
n(\%)\end{array}$ & $\begin{array}{l}\text { DHs }(N=63) \\
n(\%)\end{array}$ & $\begin{array}{l}\text { RHs and tertiary } \\
\text { hospitals }(N=17) \\
n(\%)\end{array}$ \\
\hline \multicolumn{4}{|l|}{ BEmONC } \\
\hline 1. Give parenteral antibiotic & $17(32.1)$ & $63(100.0)$ & $17(100.0)$ \\
\hline 2. Give parenteral uterotonics & $53(100.0)$ & $63(100.0)$ & $17(100.0)$ \\
\hline 3. Give parenteral anticonvulsants & $53(100.0)$ & $63(100.0)$ & $17(100.0)$ \\
\hline 4. Manual removal of retained placenta & $37(69.8)$ & $59(93.7)$ & $17(100.0)$ \\
\hline 5. Manual vacuum aspiration/D\&C & $1(1.9)$ & $53(84.1)$ & $17(100.0)$ \\
\hline 6. Assisted delivery & $2(3.8)$ & $44(69.8)$ & $15(88.2)$ \\
\hline 7. Bag-and-mask ventilate a neonate & $44(83.0)$ & $62(98.4)$ & $17(100.0)$ \\
\hline \multicolumn{4}{|l|}{ CEmONC } \\
\hline 8. Perform CS & NA & $48(76.2)$ & $17(100.0)$ \\
\hline 9. Provide blood transfusion & NA & $63(100.0)$ & $17(100.0)$ \\
\hline
\end{tabular}

Table 5. Referral policy

\begin{tabular}{llll}
\hline & $\begin{array}{l}\text { CHCs } \\
\boldsymbol{n}(\%)\end{array}$ & $\begin{array}{l}\text { DHs } \\
\boldsymbol{n}(\%)\end{array}$ & $\begin{array}{l}\text { RHs and tertiary hospitals } \\
\boldsymbol{n}(\%)\end{array}$ \\
\hline Prescribed referral routes & $43(81.1)$ & $52(82.5)$ & $16(94.1)$ \\
Prescribed referral criteria & $49(92.5)$ & $57(90.5)$ & $16(94.1)$ \\
Documented referral policies & $37(69.8)$ & $39(61.9)$ & $11(64.7)$
\end{tabular}

Table 6. Ability of $\mathrm{CHCs}$ to provide BEmOC, and distance to nearest referral hospital

\begin{tabular}{lllllll}
\hline & \multicolumn{5}{c}{ Number of signal functions, $\boldsymbol{n}$ CHCs (\%) } & \\
\cline { 2 - 6 } Distance $(\mathbf{k m})$ & $\mathbf{2}$ & $\mathbf{3}$ & $\mathbf{4}$ & $\mathbf{5}$ & $\mathbf{6}$ & Total \\
\hline$<21$ & $2(8.0)$ & $4(16.0)$ & $7(28.0)$ & $11(44.0)$ & $1(4.0)$ & 25 \\
$21-50$ & $1(5.6)$ & $4(22.2)$ & $4(22.2)$ & $9(50.0)$ & - & 18 \\
$51-75$ & - & $2(22.2)$ & $1(11.1)$ & $4(44.4)$ & $2(22.2)$ & 9 \\
$76-100$ & - & $1(100.0)$ & - & - & - & 1 \\
Total & 3 & 11 & 12 & 24 & 3 & 53
\end{tabular}

Thirty per cent of CHCs and $6 \%$ of DHs could not perform manual removal of a retained placenta at all times, but in some $\mathrm{CHCs}$ the ability to manually remove the placenta was patchy, with only $58.5 \%$ (31) having midwives capable of manually removing a placenta available at all times. Only 6 CHCs had elbow-length gloves available for manual removal of the placenta. Ninety-eight per cent and $96 \%$ of the CHCs could not perform an assisted delivery and manual vacuum aspiration for spontaneous incomplete miscarriage, respectively. Sixteen per cent of the DHs could not manage spontaneous incomplete miscarriages and 30\% could not perform assisted deliveries. Surprisingly, $17 \%$ of the $\mathrm{CHCs}$ could not bag-and-mask ventilate neonates. (This was also the case in one $\mathrm{DH}$, but that hospital could intubate a neonate.) Twenty-four per cent of the DHs could not perform CSs, but all had blood available to give blood transfusions.

Table 5 indicates whether the institutions had prescribed referral routes, criteria for referral laid down and a written policy on referral. Most health institutions had clear referral routes and criteria for referral; however, fewer had these documented.

Table 6 shows the ability of CHCs to provide $\mathrm{BEmONC}$ and the distance from their referral hospital. Over half (53\%) of the CHCs were more than $20 \mathrm{~km}$ from their nearest referral hospital, and of these 1 could provide only two signal functions, 7 only three functions and 6 only four functions, i.e. almost a third of $\mathrm{CHCs}$ were providing 
Table 7. Ability of DHs to provide CEmONC, and distance to nearest referral hospital

\begin{tabular}{lllllll}
\hline & \multicolumn{5}{c}{ Number of signal functions, $\boldsymbol{n}$ DHs (\%) } & \\
\cline { 2 - 6 } Distance $(\mathbf{k m})$ & $\mathbf{5}$ & $\mathbf{6}$ & $\mathbf{7}$ & $\mathbf{8}$ & $\mathbf{9}$ & Total \\
\hline$<21$ & - & - & $1(14.3)$ & $2(28.6)$ & $4(57.1)$ & 7 \\
$21-50$ & - & $1(16.7)$ & $2(33.3)$ & $1(16.7)$ & $2(33.3)$ & 6 \\
$51-75$ & - & $1(7.7)$ & $2(15.4)$ & $4(30.8)$ & $6(46.2)$ & 13 \\
$76-100$ & $1(8.3)$ & - & $2(16.7)$ & $4(33.3)$ & $5(41.7)$ & 12 \\
$101-150$ & $1(7.1)$ & - & - & $8(57.1)$ & $5(35.7)$ & 14 \\
$151-200$ & - & $1(10.0)$ & - & $2(20.0)$ & $7(70.0)$ & 10 \\
$>250$ & - & - & - & - & $1(100.0)$ & 1 \\
Total & 2 & 3 & 7 & 21 & 30 & 63
\end{tabular}

Table 8. Availability of quality improvement committee and conduct of maternal and perinatal death and clinical audit by type of healthcare facility

\begin{tabular}{llll}
\hline & $\begin{array}{l}\text { CHCs } \\
\boldsymbol{n}(\%)\end{array}$ & $\begin{array}{l}\text { DHs } \\
\boldsymbol{n}(\%)\end{array}$ & $\begin{array}{l}\text { RHs and tertiary hospitals } \\
\boldsymbol{n}(\%)\end{array}$ \\
\hline Quality improvement committee & $35(66.0)$ & $56(88.9)$ & $17(100.0)$ \\
Maternal death reviews conducted & $37(69.8)$ & $53(84.1)$ & $17(100.0)$ \\
Perinatal death reviews conducted & $47(88.7)$ & $62(98.4)$ & $16(94.1)$ \\
Process clinical audits & $33(62.3)$ & $52(82.5)$ & 1376.5
\end{tabular}

four or fewer signal functions. Table 7 shows that $7 \mathrm{DHs}$ were providing seven or fewer signal functions and were $50 \mathrm{~km}$ or more from an RH. Fifteen DHs did not provide a CS service and 14 (93\%) were more than 20 $\mathrm{km}$ from an $\mathrm{RH}$.

The sites were asked to comment on their referral system. The most frequent comment was delays in the ambulance service (25, 40\%).

Table 8 gives the performance of quality improvement activities and audits at the various facilities.

\section{Discussion}

This survey was undertaken to assess the ability of health facilities in 12 districts to provide emergency obstetric care. It is the first survey of its kind to be undertaken in SA, although similar surveys have been conducted in other countries. Overall no CHC could provide all seven BEmONC signal functions. Forty-nine per cent of the CHCs could perform only four signal functions, and $25 \%$ were able to perform only three signal functions. Most CHCs could not perform an assisted delivery or manual vacuum aspiration for a spontaneous incomplete miscarriage, but all had magnesium sulphate and oxytocics. Fortyeight per cent of the DHs could perform all nine of the CEmOC signal functions, and altogether $81 \%$ could perform eight of the nine signal functions. The most common signal functions that the DHs were not able to perform were assisted delivery (30\%), CS (24\%) and manual vacuum aspiration for a spontaneous incomplete miscarriage (16\%).

Surprisingly few (32\%) of the CHCs could give parenteral antibiotics. This could be explained by the earlier versions of the Essential Drugs List not containing intravenous antibiotics for CHCs. This has since changed, and the Essential Drugs List now includes parenteral antibiotics for CHCs. Starting intravenous antibiotics as soon as possible in cases of pregnancy-related sepsis or septic miscarriage improves the chances of women with these complications surviving. Most CHCs had oral antibiotics, but this would be inadequate for initiating treatment in cases of severe sepsis.

Obstetric haemorrhage, especially postpartum haemorrhage (PPH), is unpredictable and death can occur rapidly. It has been reported that in the absence of any medical intervention the average time to death from a $\mathrm{PPH}$ is 2 hours and that for an antepartum haemorrhage 12 hours. In the Birthplace in England Collaborative Group study on birthplaces for women classified as having low-risk pregnancies, $1.2 \%$ of women ended up requiring blood transfusion for obstetric haemorrhage. ${ }^{[4]}$ It is essential for all sites conducting births to have the ability (knowledge, skills and resources) to manage PPH. Furthermore, it is important for all sites to practise the active management of the third stage of labour, which includes giving oxytocin to all women in the third stage of labour. A retained placenta is a common cause of severe $\mathrm{PPH}$, and the sixth Saving Mothers report indicated that 45 women died of this complication ( $7 \%$ of all maternal deaths due to obstetric haemorrhage). ${ }^{[2]}$ Manual removal of a placenta is an essential skill that all healthcare providers conducting births should have. Thirty per cent of CHCs and 6\% of DHs did not have anyone with the ability to perform this function. This indicates a deficit in the health system. Furthermore, in only $31 \mathrm{CHCs}(58 \%)$ were there midwives capable of manually removing a placenta, suggesting that this skill is not available around the clock in CHCs. The almost total lack of appropriate gloves for the procedure (47/53 CHCs) indicates a lack of knowledge about the appropriate equipment for this procedure.

Administering magnesium sulphate to all women with eclampsia and severe preeclampsia as soon as the condition is recognised is one of the most important ways of reducing deaths due to complications of hypertension. To administer magnesium sulphate safely and give a repeat dose, the healthcare provider needs to be able to test the patient's reflexes, and to administer calcium gluconate if there is an overdose of magnesium sulphate.

Left unmanaged, spontaneous incomplete miscarriages (which occur in $15 \%$ of women who are clinically pregnant) have a significant risk of becoming infected or resulting in severe blood loss. In $2011-2013,{ }^{[2]} 114$ and 48 women died of sepsis or haemorrhage following a miscarriage, respectively. Much of this can be prevented if the uterus can be evacuated as soon as possible. The manual vacuum aspirator (MVA) is a simple piece of apparatus that enables a woman with an uncomplicated incomplete miscarriage to undergo evacuation of the uterus as an outpatient. In a randomised trial it was shown to be superior to an evacuation in theatre ${ }^{[9]}$ It is surprising that use of an MVA was not available at $27 \mathrm{DHs}$ and $5 \mathrm{RHs}$, and they were almost absent from the CHCs. All doctors should be skilled in the technique. In SA all midwives with advanced midwifery, and specially trained midwives performing terminations of pregnancy, are trained and legislated to perform this procedure.

In the Birthplace in England Collaborative Group study on perinatal outcomes of low-risk pregnancies, ${ }^{[4]} 7.3 \%$ and $6.2 \%$ of women had vacuum and forceps deliveries, respectively. The perinatal mortality rate (PNMR) in this population was $<1 / 1000$ births. In SA, the PNMR for babies weighing $\geq 1000 \mathrm{~g}$ was $\sim 25.6 / 1000$ births for $2010-2011 .^{[11]}$ Intrapartum asphyxia and birth trauma were the major underlying causes of perinatal death, with a rate of 4.87/1 000 births. Our assisted delivery rate 
was $0.52 \%$ for vacuum delivery and $0.15 \%$ for forceps delivery. The CS rate was $21 \%$. When the intrapartum asphyxia and birth trauma death rates were correlated with the vacuum delivery rate, there was a significant negative correlation of $r=-0.307(p=0.036){ }^{[10]}$

A negative correlation means that the fewer vacuum-assisted deliveries there were, the more intrapartum asphyxia and birth trauma deaths occurred. An assisted delivery rate of $<1 \%$ is too low, and is probably due to the loss of skill in performing assisted delivery. Even at the tertiary level there is lack of ability to perform assisted delivery. Assisted delivery is a key activity to reduce perinatal deaths by reducing delay in delivery and is an important function for CHCs. All advanced midwives should be able to perform at least a vacuum delivery. The fact that these skills are almost totally absent in the CHCs speaks for itself and must contribute to the high mortality rate due to intrapartum asphyxia and birth trauma.

About 3\% of babies born in the Birthplace in England Collaborative Group study required neonatal resuscitation. ${ }^{[4]}$ It is always possible that a neonate will need to be resuscitated, and every healthcare provider conducting births must be able to at least bag-and-mask ventilate a neonate. That $17 \%$ of $\mathrm{CHCs}$ were not able to do so owing to lack of equipment or skills is very disturbing.

Unexpectedly, 15 (24\%) of the DHs in the 12 districts were not performing CSs. The most common reasons were that there were no doctors (11/15) and no functioning theatre (4/15). However, all DHs could give a blood transfusion if necessary. Surprisingly, $16 \%$ of DHs did not have maternal death reviews.

Recently Dumont et al. ${ }^{[1]}$ in the QUARITE trial demonstrated that an intervention consisting of emergency obstetric care training and training in conducting maternal mortality review meetings, with monthly meetings, combined with continuing medical education on the problems identified, reduced maternal mortality by $15 \%$. Both these activities (emergency obstetric care training and maternal mortality review meetings) are being scaled up in SA. However, if increased knowledge and skills are not accompanied by increased functionality of the facilities with respect to lifesaving services and effective interfacility transport, the impact of the increased knowledge and skills may be smaller than expected.

The WHO Multicountry Survey on Maternal and Newborn Health analysed the use of the signal function in a random sample of larger hospitals ( $>1000$ births per year) and found surprisingly high mortality rates. ${ }^{[12]}$ They concluded that having the ability to provide lifesaving services is only useful if healthcare providers are skilled in the management of complicated cases. If the healthcare providers are not skilled in managing a complication the result will be poor, even if the resources are available to manage the case. In the 12 districts studied we do not have good coverage of the basic and comprehensive emergency care signal functions, and the high number of cases that were thought to be possibly or probably avoidable in the Saving Mothers reports would indicate that we also lack the skills to manage complications.

This study concentrates on the 12 most needy districts in SA, so the picture represented here is the worst-case scenario. A survey of other districts needs to be performed to see whether the problems identified in this study are present in all the districts.

A feature not discussed in this paper is the staffing of the various facilities. This is dealt with in a separate paper. ${ }^{[13]}$

A number of recommendations have evolved out of this survey. The CEOs of the institutions and the district managers must be made aware of the requirements of providing the seven signal functions for $\mathrm{BEmONC}$, and that all DHs should be able to provide the nine signal functions for CEmONC.

Currently the scope of practice of a professional nurse with midwifery (the vast majority of nursing staff running maternity units) does not allow them to perform assisted delivery and manual vacuum aspiration. All advanced midwives should have the knowledge and skills to perform vacuum deliveries, manual vacuum aspiration for incomplete miscarriages and manual removal of the placenta. A method must be found to train advanced midwives rapidly to fill the skills gap, especially in CHCs.

Special attention needs to be paid to the sites performing CSs. There need to be adequate skills and facilities to provide the service safely.

A concerted effort must be made to ensure that advanced midwives and doctors are skilled in vacuum deliveries, manual removal of the placenta and manual vacuum aspiration of the uterus for incomplete miscarriage. (Training in these skills is part of the ESMOE-EOST programme.)

CEOs of $\mathrm{CHCs}$ and DHs need to ensure that they employ healthcare providers who provide the correct skills mix to ensure that basic and comprehensive emergency care can be effectively provided. This area is one where task shifting can be fruitfully employed.

Special attention needs to be paid to improving interfacility transport. Use of the Free State model of separating the prehospital and interfacility ambulance service could be a very effective route to follow. ${ }^{[14]}$

\section{Conclusion}

In the 12 districts surveyed, the required level of emergency obstetric care was not available in most CHCs and in a quarter of DHs. If referral of patients to an appropriate level is taken into consideration, less than half the CHCs could possibly get their patients with complications to the appropriate hospital in less than 1 hour.

The ability of the primary level of care (CHCs and DHs) to perform the signal functions (lifesaving services) of basic and comprehensive emergency obstetric care was poor in many of the districts studied. This implies that safe maternity care was not consistently available at many facilities conducting births.

Acknowledgements. The authors thank Dr Charles Ameh for input on the baseline datasheet. We would also like to thank all the district managers, CEOs of facilities and staff who we interviewed and who helped with the study in the 12 districts.

\footnotetext{
1. Dorrington RE, Bradshaw D, Laubscher R. Rapid Mortality Surveillance Report 2012. Cape Town: South African Medical Research Council, 2014.

2. Pattinson RC, ed. Saving Mothers 2011-2013: The Sixth Report of the National Committee for Confidential Enquiries into Maternal Deaths in South Africa. Pretoria: Government Printer, 2014. Confidential Enquiries into Maternal Deaths in South Africa. Pretoria: Government Printer, 2014.
Rooks JP, Weatherby NL, Ernst EK, Stapleton S, Rosen D, Rosenfield A. Outcomes of care in birth 3. Rooks JP, Weatherby NL, Ernst EK, Stapleton S, Rosen D, Rosenfield A. Outcomes of care in birth
centers: The National Birth Center Study. N Engl J Med 1989;321(26):1804-1811. [http://dx.doi. org/10.1056/NEJM198912283212606]

4. Birthplace in England Collaborative Group. Perinatal and maternal outcomes by planned place of birth for healthy women with low risk pregnancies: The Birthplace in England national prospective cohort study. BMJ 2011;343:d7400. [http://dx.doi.org/10.1136/bmj.d7400]

5. Kerber K. Triple return for our rand: How many South African mothers and babies can be saved and what is the cost? Presented at the 30th Annual Priorities in Perinatal Care Conference, Safari Lodge, Polokwane, 8-10 March 2011.

6. Pattinson R, Kerber K, Buchmann E, et al., for The Lancet's Stillbirths Series steering team. Stillbirths: How can health systems deliver for mothers and babies? Lancet 2011;377(9777):1610-1623. [http:// dx.doi.org/10.1016/S0140-6736(10)62306-9]

7. World Health Organization. Monitoring Emergency Obstetric Care: A Handbook. Geneva: WHO, 2009.

8. Frank K, Lombaard H, Pattinson RC. Does completion of the Essential Steps in Managing Obstetric Emergencies (ESMOE) training package result in improved knowledge and skills in managing
Eng Emergencies (ESMOE) training package result in improved k

obstetric emergencies? S Afr J Obstet Gynaecol 2009;15(3):94-99.
9. De Jonge ETM, Venter CP, de Wet GH, Pattinson RC. Improving care for patients undergoing curettage 9. De Jonge ETM, Venter CP, de Wet GH, Pattinson RC. Improvige
for incomplete abortion. S Afr Med J 1996;86(9):1172-1174.

10. Pattinson RC. Saving Babies 2010-2011: The Eighth Report on Perinatal Care in South Africa. Pretoria: Tshepesa Press, 2012

11. Dumont A, Fournier P, Abrahamowicz M, et al. Quality of care, risk management, and technology in obstetrics to reduce hospital-based maternal mortality in Senegal and Mali (QUARITE): A clusterrandomised trial. Lancet 2013;382(9887):146-157. [http://dx.doi.org/10.1016/S0140-6736(13)60593-0]

12. Souza JP, Gülmezoglu AM, Vogel J, et al. Moving beyond essential interventions for reduction of maternal mortality (the WHO Multicountry Survey on Maternal and Newborn Health): A crosssectional study. Lancet 2013;381(9879):1747-1755. [http://dx.doi.org/10.1016/S0140-6736(13)60686-8]

13. Pattinson RC Safety versus accessibility in maternal and perinatal care. S Afr Med J 2015:105(4) 261265. [http://dx.doi.org/10.7196/SAMJ 9182$]$

4. Schoon M. Impact of inter-facility transport on maternal mortality in the Free State Province. S Afr 4. Schoon M. Impact of inter-facility transport on maternal mortality in
Med J 2013;103(8):534-537. [http://dx.doi.org/10.7196/samj.6828]

5. World Health Organization. Managing Complications in Pregnancy and Childbirth: A Guide for Midwives and Doctors. (WHO/RHR/00.7). Geneva: WHO, Geneva, 2003.
}

Accepted 24 November 2014. 